\title{
Biología floral de MAMMILLARIA HUITZILOPOCHTLI, UNA ESPECIE RARA QUE HABITA ACANTILADOS
}

\author{
Alejandro Flores-Martínez ${ }^{1}{ }^{1,2}$, Gladys I. Manzanero M. ${ }^{1}$, Jordan Golubov ${ }^{2}$ \\ y María C. ManduJANO 3,4 \\ ${ }^{1}$ Centro Interdisciplinario de Investigación para el Desarrollo Integral Regional, \\ Instituto Politécnico Nacional Santa Cruz Xoxocotlán, Oaxaca, México \\ ${ }^{2}$ Universidad Autónoma Metropolitana Xochimilco. México, D.F. México \\ ${ }^{3}$ Depto. Ecología de la Biodiversidad, Instituto de Ecología, \\ Universidad Nacional Autónoma de México, México, D.F., México \\ † in memoriam \\ ${ }^{4}$ Autor para la correspondencia: mcmandu@ecologia.unam.mx
}

\begin{abstract}
Resumen: La producción de semillas y el reclutamiento en las especies dependen en parte de factores reproductivos, tales como el comportamiento floral, la fenología de la floración, la producción de gametos y néctar, los sistemas de cruzamiento y de apareamiento, y la densidad poblacional. En este trabajo se estudia la biología floral, producción de néctar y polinización de Mammillaria huitzilopochtli D.R.Hunt en condiciones ex situ para sentar las bases que pueden ayudar a determinar el riesgo de extinción de la especie dado su sistema de apareamiento y éxito reproductivo. La floración de $M$. huitzilopochtli abarca cuatro meses (septiembre a diciembre), de antesis diurna, con longevidad por flor de alrededor de seis días, se presentó baja la producción de néctar (2.02 $\mu$ l) y éste sólo se detectó en el 39\% de las flores. La especie presenta un sistema de entrecruza xenógamo facultativo, con una relación polen:óvulo de 1,048. La producción de semillas por fruto es variable y baja con relación al número de óvulos promedio (35\%). Existen muy pocos trabajos de biología floral en el género Mammillaria, pero al compararla con otras cactáceas, el comportamiento floral es similar a las especies de polinización melitófila como Echinocactus, Grusonia y Opuntia. La longevidad floral es la más prolongada de las reportadas y, el dimorfismo en la producción de néctar y el asentamiento de frutos vacíos no se había registrado para esta especie. El sistema de apareamiento xenógamo facultativo y el entrecruzamiento son frecuentes en las cactáceas raras y en peligro de extinción. Su condición de xenógama, la amplia varianza en el número de semillas por fruto y la incapacidad para clonar incrementa su riesgo.
\end{abstract}

Palabras clave: biología floral, cactáceas, especies raras, sistemas de cruzamiento.

\begin{abstract}
Seed production and the recruitment of new individuals are in part related to reproductive traits such as floral behaviour, phenology, production of sexual gametes, nectar, mating and breeding systems, and population densities. We studied the floral biology, nectar production, and pollination of Mammillaria huitzilopochtli D.R.Hunt in ex situ conditions to provide support that can be used to determine extinction risk of the species given its breeding system and reproductive success. Flowering of $M$. huitzilopochtli covers a four month period (September to December), with diurnal anthesis and a floral longevity of around six days. Nectar production was low $(2.02 \mu \mathrm{l})$ and found in $39 \%$ of sampled flowers. The species presents a facultative outcrossing breeding system, with a ovule:polen ratio of 1,048 . Seed set was highly variable and low (35\%) if compared with the mean number of ovules. There are few treatments on floral biology of Mammillaria, but comparing with other Cactaceae, floral behaviour was similar to other bee pollinated species such as Echinocactus, Grusonia, and Opuntia. Floral longevity is longer than reported for other Cactaceae and both the presence of dimorphic nectar production and empty fruits had not been reported previously. The facultative xenogamous breeding system and outcrossing mating system are common in rare cactus species. This condition coupled with the variation in seed set and lack of clonal propagation adds factors towards risk.
\end{abstract}

Key words: breeding systems, cacti, floral biology, rarity.

$\mathbf{L}$ as cactáceas que habitan en zonas áridas generalmente presentan poco (o nulo en algunos años) reclutamiento de nuevos individuos por vía sexual (Franco y Nobel, 1989;
Mandujano et al., 2001; 2007). Este fenómeno puede deberse a una limitada o insuficiente producción de semillas relacionada con varios aspectos del sistema reproductivo. 
La cantidad de semillas producidas puede depender del sistema de cruzamiento, del sistema de apareamiento y de la densidad poblacional (Schemske et al., 1994; Kunin, 1997; Gigon et al., 2000), así como del número de óvulos disponibles y la probabilidad de su polinización (Orians, 1997). Además, los factores como la composición genética de la especie, ausencia o ineficiencia de polinizadores, también juegan un papel importante en el éxito reproductivo (Mandujano et al., 1996; Gaston y Kunin, 1997; Kunin, 1997; Orians, 1997; Pimienta-Barrios y del Castillo, 2002). Las principales causas del bajo reclutamiento pueden analizarse por medio del estudio de la biología floral de estas especies, ya que permite conocer el papel de los factores proximales que afectan la producción de semillas.

Algunos autores proponen tendencias evolutivas de las especies raras de acuerdo a factores de la biología floral, como son el sistema de cruza y el sistema de apareamiento (Richards, 1986; Martínez-Peralta y Mandujano, 2011). Existen tres tipos principales de sistemas de cruza: autocruza, entrecruza y mixto; y cinco tipos de sistemas de apareamiento con relación al cociente polen:óvulo, que son cleistógamo, autógamo (obligado o facultativo) y xenógamo (obligado o facultativo; Cruden, 1977). En las especies raras se propone que los sistemas de apareamiento de autogamia se presentan cuando es baja la densidad poblacional; en contraste, la xenogamia se presenta con altas densidades poblacionales (Karron, 1997). Otros autores sugieren que las especies raras tienen mecanismos que incrementan el número de polinizadores si su sistema de apareamiento es xenógamo mediado por polinizadores generalistas, como el número de flores abiertas por día, tiempo de vida de cada flor y cantidad de polen o néctar, entre otros (Gaston y Kunin, 1997; Orians, 1997).

El tipo de sistema de apareamiento de la especie se determina de manera indirecta con el número de óvulos y granos de polen por flor, así como el número de frutos y semillas producidas en experimentos de polinización controlada (Dafni, 1992; Kearns e Inouye, 1993; Pimienta-Barrios y del Castillo, 2002). Estas variables aportan evidencia en torno al tipo de apareamiento predominante. Cruden (1977) usó la relación polen:óvulo, bajo el supuesto de que las especies que son autógamas no necesitan producir una gran cantidad de polen, mientras que las especies xenógamas sí lo requieren, para estimar el sistema de apareamiento. Dafni (1992) utiliza un índice que mide la tasa de incompatibilidad (Incompatibility System Index, ISI) por medio de la proporción del número de frutos producidos por autocruza y el número de frutos producido por entrecruza.

Dentro de los atributos florales que determinan la eficiencia del sistema reproductivo está la producción de néctar. Algunos autores sugieren que, considerando que el néctar es una sustancia que no forma parte del sistema reproductivo de la planta y que sólo es una recompensa para los polinizadores, puede existir una correlación entre la cantidad y ritmo de producción de néctar con la demanda y comportamiento de los polinizadores: si el néctar es muy importante en la interacción planta-polinizador, es de esperarse una relación coevolutiva entre ellos, lo que induce a que las características del néctar tiendan a ser similares cuando las plantas tienen polinizadores específicos de taxa similar (Dafni, 1992; Kearns e Inouye, 1993).

Por otro lado, el efecto que tiene la densidad poblacional en el tipo de sistema de cruzamiento de especies raras casi no se ha estudiado experimentalmente. Kunin (1997) y Orians (1997) mencionan una posible tendencia de las especies raras hacia la autogamia a que se vea favorecida la ocurrencia de mecanismos de reproducción clonal debida a su baja densidad (lo cual no atrae polinizadores); sin embargo, esta tendencia no siempre se cumple debido a que la rareza no depende solamente del factor densidad (Rabinowitz et al., 1986). Algunos estudios sugieren que las especies raras que tienen xenogamia obligada (y polinizadas por insectos), están relacionadas positivamente con la densidad poblacional, ya que los insectos pueden hacer los recorridos entre plantas, lo que reduce el gasto energético, mientras que a bajas densidades poblacionales puede predominar la autopolinización (en la que no intervienen los insectos) o la geitonogamia (Orians, 1997). Otros estudios sugieren que algunos tipos de especies raras con baja abundancia, densidad o área de distribución, generalmente no tienen especies florívoras asociadas a ellas, lo que permite que se presente una tendencia hacia flores de larga vida, polinizadores generalistas y una menor eficiencia en la transferencia de polen (Karron, 1997; Orians, 1997).

Mammillaria huitzilopochtli es una cactácea globosa endémica del Valle de Cuicatlán. La especie está considerada como "sujeta a protección especial" (Pr) en la Norma Oficial Mexicana (NOM-059-SEMARNAT-2001; SEMARNAT, 2002). La densidad poblacional de la especie va desde $3,850 \mathrm{ind}$./ha hasta $900 \mathrm{ind}$./ha, y su actual área de distribución está sujeta a diferentes grados de perturbación humana. En este trabajo se estudió su biología floral para determinar los factores que influyen en la producción de semillas y garantizan el reclutamiento de nuevos individuos. Con este objetivo se determinó el comportamiento floral, la fenología de la floración, producción de óvulos, polen y néctar, y se evaluaron los sistemas de cruzamiento y de apareamiento de la especie. Los datos de este estudio también permiten sugerir la posibilidad de una relación con algunas variables poblacionales (como su bajo nivel de reclutamiento o producción insuficiente de semillas), así como relacionar estos comportamientos florales como consecuencia de su rareza y vulnerabilidad.

\section{Métodos}

Comportamiento floral y fenología de la floración. Los individuos usados en el estudio fueron plantas reproducti- 
vas establecidas en el vivero del Jardín Botánico Regional "Cassiano Conzatti" del CIIDIR (Centro Interdisciplinario de Investigación para el Desarrollo Integral Regional) IPN Unidad Oaxaca (JB). Las plantas se colocaron al exterior para permitir la llegada de polinizadores. Todas son plantas procedentes de dos poblaciones de estudio de Mammillaria huitzilopochtli y tienen tamaños que corresponden a los adultos maduros y viejos desde 4.1 a $10 \mathrm{~cm}$ de altura. En esta especie, las categorías reproductivas se inician de tallas muy pequeñas $(2 \mathrm{~cm}$ de altura) y el número de estructuras reproductivas decrece con el tamaño (menor en adultos de 7 a $10 \mathrm{~cm}$ de altura). Independientemente del tamaño, la contribución percápita al reclutamiento de plántulas, dada por un individuo reproductivo a lo largo de su vida en las diferentes categorías de ciclo de vida, es reducida (Flores Martínez et al., 2010). Las poblaciones de origen están localizadas en el Valle de Cuicatlán, en un sitio perturbado (PDS, $17^{\circ} 48^{\prime} 41^{\prime \prime}$ latitud norte, 96 $56^{\circ}$ ' $50^{\prime}$ longitud oeste, en altitudes de 750 a 900 m s.n.m.), y otra población establecida en un sitio más conservado (PCS, $17^{\circ} 54^{\prime} 19^{\prime \prime}$ latitud norte, $96^{\circ}$ 57' 56" longitud oeste, en altitudes de 600 a 750 m s.n.m., Flores Martínez et al., 2010). Las plantas, en condiciones naturales, se encuentran en pendientes pronunciadas, por lo que la manipulación y observación se hizo ex situ. Las flores de cada individuo se marcaron, se registró en cada caso la fecha de apertura de la flor, fecha de inicio y término de producción de polen y de receptividad del gineceo, y la fecha del cierre de la flor. Cada día también se registró el tiempo de apertura de las flores. Con estos datos se estimó el tipo de apertura de flor (diurna o nocturna), la longevidad floral (en días) por flor y por individuo. La producción total de flores y el tiempo de duración del periodo de floración por individuo se estimó en el año $2003(N=7)$ y en el $2006(N=9)$. Diferencias entre la duración de flores por individuo y la producción de flores por individuo entre años se analizaron por medio de Kruskal-Wallis, además de una correlación entre la producción de flores (número de flores) y el periodo de floración (en días) de cada planta.

Recompensas florales: producción de néctar. La producción de néctar se midió en 82 flores embolsadas, elegidas al azar de las plantas establecidas en el vivero $(N=7$, plantas en 2003 y $N=9$ en 2006). Estas flores ya se encontraban en su fase de producción de polen (con uno a dos días de vida). Para la medición del volumen de néctar se utilizaron micropipetas con volumen de $2 \mu \mathrm{l}$. El volumen de néctar se midió un día por flor, ya sea el primer o segundo día de vida de la flor, dependiendo del inicio de la fase de producción de polen, entre las 13:00 y las 15:00 h. La medición de néctar se efectuó utilizando el método del volumen acumulado (una sola toma de néctar $N=74$ flores). Una prueba piloto sugería la ausencia de reemplazo de néctar, pero también se incluyó una prueba seriada de producción de néctar (en tres ocasiones 10:00, 13:00 y 15:00 h durante el mismo día, $N=$ 8 flores únicamente en 2003; Kearns e Inouye, 1993).

Sistema de apareamiento. Para evaluar el sistema de apareamiento (Cruden, 1977) se utilizaron el número de óvulos, anteras y granos de polen por flor $(N=10$ flores al azar en cuatro individuos) en flores excluidas de polinizadores durante la floración de 2004. Con estos datos se determinó el promedio de óvulos por flor y la relación polen:óvulo. Cada ovario y antera se incluyeron en $2 \mathrm{ml}$ de solución jabonosa. Una vez en suspensión, las estructuras se contaron en hemacitómetro de una muestra de $25 \mu \mathrm{l}$. El total de granos de polen fue calculado a partir del número de anteras por flor, así como por el factor de dilución (Kearns e Inouye, 1993). Finalmente se estimó la proporción polen:óvulo (Cruden, 1977) para determinar el sistema de apareamiento.

Sistema de cruzamiento. La evaluación del sistema de cruzamiento se hizo usando cruzas controladas con cuatro tratamientos (autocruza forzada, autocruza natural, geitonogamia y entrecruza forzada), y un control (entrecruza natural). Los tratamientos se aplicaron a 229 flores reproductivas $(N=13)$, establecidas en el vivero del JB en 2005. En los tratamientos de autocruza forzada, geitonogamia y entrecruza forzada se cortaron estambres con polen maduro para su aplicación en el estigma receptivo de la flor: en el caso de autocruza forzada $(N=15)$ el polen de la misma flor, en el caso de las cruzas geitonogámicas $(N=15)$ el estambre fue de otra flor pero de la misma planta, y en el caso de la entrecruza forzada $(N=3)$ el estambre era de otra flor y de otro individuo. El estigma se consideró como receptivo cuando estaba abierto y con superficie pegajosa (los lóbulos del estigma se separan o abren de manera similar a la apertura de los segmentos del perianto; varios días el estigma permanece cerrado). Una vez efectuadas las cruzas, las plantas fueron emasculadas de su porción masculina y cubiertas con bolsas de tul para evitar la entrada de polinizadores. La hora promedio en la que se polinizaron las flores fue a las 13:00 $\mathrm{h}$.

En las flores donde se evaluó la autocruza natural (no forzada) $(N=196)$, para impedir la acción de los polinizadores las plantas fueron cubiertas con bolsas de tela fina de mosquitero antes de iniciar el periodo de floración. La evaluación de la cruza natural (control) se efectuó en los frutos de los individuos del JB que se encontraban en la colección de plantas vivas y expuestas al aire libre, y a posibles polinizadores ( $N=81$ frutos en 13 plantas). Finalmente, se hizo una comparación de los promedios de semillas de los frutos colectados en campo de las poblaciones establecidas en el Valle de Cuicatlán in situ, con los provenientes de la colección del JB. En todos los tipos de cruzas se revisó si había producción de frutos. Los frutos formados se colectaron y se contó el número de semillas.

Se calculó el índice de incompatibilidad (ISI) por medio de la relación entre el número de frutos producidos por auto- 
fecundación dividido entre el número de frutos producidos por entrecruza, donde un valor menor a 0.2 implica una especie "casi” xenógama obligada (Dafni, 1992).

\section{Resultados}

Comportamiento floral y fenología de la floración. Mammillaria huitzilopochtli florece una vez al año, en los meses de septiembre a diciembre. Las flores son hermafroditas ciatiformes homógamas y diurnas con hora de apertura variable, principalmente entre las 10:00 y las 12:00 horas, y cierran entre las 17:00 a 19:00 horas. La longevidad de las flores varía de uno hasta más de seis días. El análisis de Kruskal-Wallis indicó que no hubo diferencia significativa entre estas longevidades promedio en ambos años (2003: $\chi^{2}=3.5263, g l=3, P=0.3175 ; 2006: \chi^{2}=4.2424, g l=3$, $P=0.2364)$. La mayoría de las flores duraron de entre 3 y 8 días (5\% de 1 a 2 días, $28 \%$ de 3 a 5 días, $50 \%$ de 6 a 8 días y el $5 \%$ más de 8 días). En todos los casos hubo producción constante de polen; sin embargo, el gineceo únicamente fue receptivo de 1 a 2 días.

La productividad de flores (número de flores producidas) y el tiempo (en días) del periodo de floración por individuo fue variable en los dos años muestreados (Figura 1), pero no se presentó una diferencia significativa entre años y la producción de flores por planta $\left(\chi^{2}=0.1811, g l=1, P=0.6704\right)$, ni en el tiempo de floración ( $\chi^{2}=0.8119, g l=1, P=0.3676$ ). Sin embargo, hubo una correlación entre el número total de
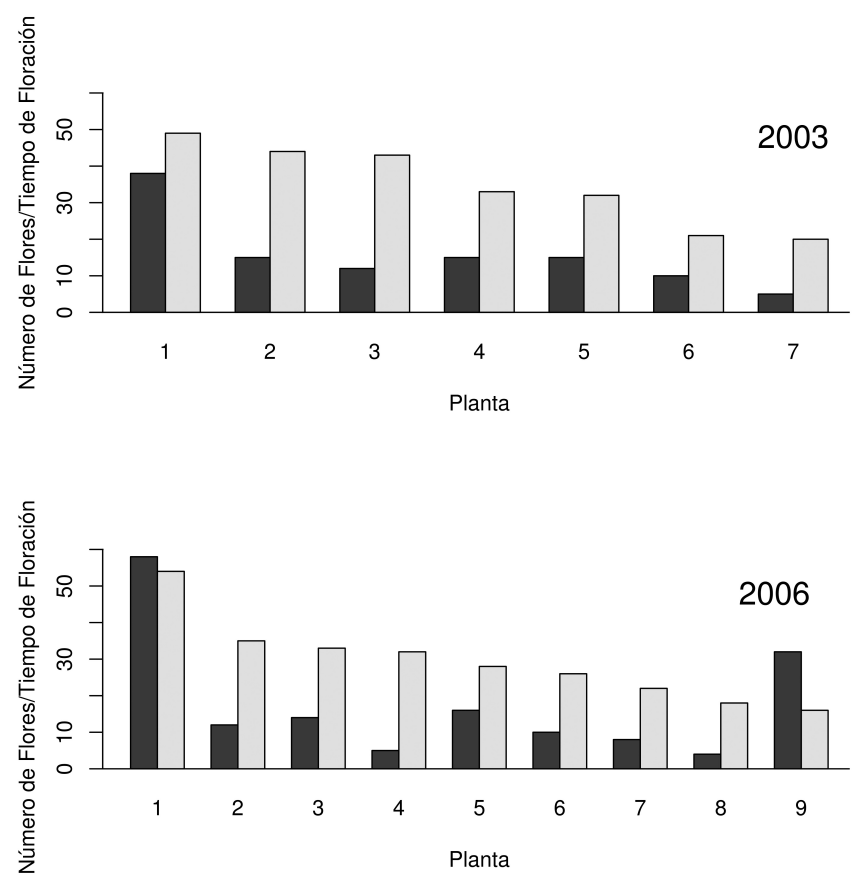

Figura 1. Número de flores producidas y tiempo de floración por planta en 2003 y 2006. Las barras oscuras indican el total de flores producidas por planta. Las barras claras indican el tiempo de floración en días. flores que produjo cada planta con el número de días que duró su periodo de floración $\left(r=0.604 ; r^{2}=0.36 ; F_{1,21}=12.08\right.$; $P=0.0023$, Figura 2). No todos los individuos tuvieron flores todos los años, independientemente de su tamaño. El amplio periodo de floración de cada planta, aunado al número de flores por planta, abre la posibilidad hacia la geitonogamia.

Recompensas florales: producción de néctar. Mammillaria huitziliopochtli tiene un sistema dimórfico de producción de néctar al nivel de individuo y al nivel de flores. Algunos individuos sólo tuvieron flores sin néctar y, en un mismo individuo se presentaron flores con y sin néctar. Sólo se encontró néctar en 39.2\% de las flores muestreadas. De las flores que tuvieron néctar, el promedio de producción en pie fue de $2.02( \pm 1.01) \mu l$. La producción de néctar al parecer no es continua, ya que en las tomas seriadas no hubo producción a lo largo del día, lo que implica la ausencia del reemplazo de néctar (nectar replenishment).

Sistema de apareamiento. Las flores tienen en promedio 92.3 anteras $( \pm 4.93, N=10)$ y un promedio de 114,523 granos de polen por flor ( $\pm 102,158 ; N=10$ flores). En contraste, la cantidad de óvulos por flor es tres órdenes de magnitud menor (promedio $=111 \pm 16.5, N=10$ ), la razón polen: óvulo estimada es de 1,048 , que corresponde a la categoría de xenógamo facultativo a xenógamo, de acuerdo a la clasificación de Cruden (1977).

Sistema de cruzamiento. En los experimentos de autocruza forzada ( $N=15$ flores, de los cuales se formaron 10 frutos) y autocruza natural $(N=196$, de los cuales se formaron 176 frutos), se presentó alta producción de frutos $(66.66 \%$ en

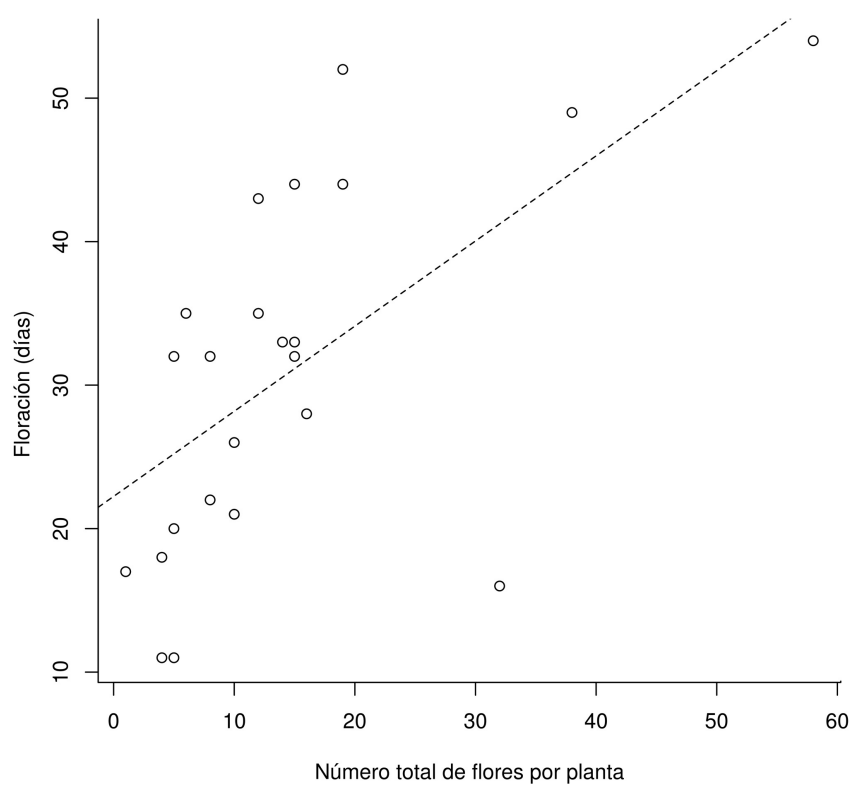

Figura 2. Relación entre el número de flores y la duración del periodo de floración por planta; datos de 2003 y 2006 ( $y=22.24+$ $\left.0.593 x ; r=0.604 ; r^{2}=0.36 ; P=0.0023\right)$. 
autocruza forzada y $89.79 \%$ en autocruza natural). Sólo dos frutos de autocruza natural tuvieron semillas $(1.13 \%)$, los frutos formados en este tratamiento contuvieron 1 y 17 semillas. En los experimentos de geitonogamia hubo producción de frutos $(N=9,60 \%)$, pero ningún fruto tuvo semillas (todos fueron frutos vanos), lo que corrobora la limitante genética de producción de semillas entre óvulos y polen del mismo individuo. En los experimentos de entrecruza forzada y cruza natural (control) todas las flores formaron frutos y tuvieron semillas, pero con amplia diferencia en el número de semillas por fruto (promedio $=38.3 \pm 38.4$ ). En relación con los frutos colectados en campo, tuvieron un menor promedio de semillas por fruto que los individuos establecidos en el jardín botánico, pero la diferencia no fue significativa $\left(\chi^{2}=1.0263, g l=1, P=0.311\right)$. El valor de ISI $(0.01)$ indica que Mammillaria huitzilopochtli es una especie casi xenógama obligada.

\section{Discusión}

En la familia Cactaceae se presentan diferentes periodos de floración. Por ejemplo, especies que florecen todo el año (p. ej. Echinocactus, Escontria, Melocactus, Praecereus, Stenocereus, Weberbauerocereus y Weberocereus; Nassar et al., 1997; Godínez-Álvarez et al., 2003; Nassar y Ramírez, 2004; Oaxaca-Villa et al., 2006; Jiménez-Sierra et al., 2007; Mandujano et al., 2010); en los meses secos del año, al inicio de la primavera (p. ej. Echinocereus pulchellus var. pulchellus, Ferocactus histrix, Mammillaria crucigera y M. oteroi (del Castillo, 1988, Contreras y Valverde, 2002; Navarro y Flores, 2002, Martínez et al., 2004), o en verano (p. ej. Carnegiea, Cephalocereus, Echinocereus, Echinomastus, Ferocactus, Hylocereus, Lophocereus, Neobuxbaumia, Opuntia, Pachycereus, Pilosocereus, Polaskia, Stenocereus; Steenbergh y Lowe, 1969; Mandujano et al., 1996; Valiente-Banuet et al., 1996, 1997; Nassar et al., 1997; Casas et al., 1999; Rojas-Martínez et al., 1999; GodínezÁlvarez et al., 2003; Mandujano et al., 2010). El período de floración de M. huitzilopochtli es a fines de la temporada de lluvias, comportamiento similar al de algunas columnares y arbustivas de los géneros Cereus, Myrtillocactus, Opuntia, Pachycereus, Pilosocereus, Selenicereus y Stenocereus (Pimienta-Barrios y del Castillo, 2002; Mandujano et al., 2010) y diferente a las Mammillaria estudiadas del Valle de Tehuacán. Este período de floración probablemente está relacionado con la cantidad de agua en los tejidos suculentos, que permite la formación de estructuras reproductivas al iniciarse la temporada de sequía (Pimienta-Barrios y del Castillo, 2002), ya que el hábitat limita la formación de suelo y la capacidad de retención de humedad.

En cactáceas, se ha reportado una amplia variación en los horarios de antesis, aparentemente relacionada con el síndrome de polinización. En Mammillaria huitzilopochtli la antesis es diurna y parece que responde a la incidencia directa de la luz solar. Comportamiento similar a la especie de este estudio en la apertura floral lo presentan algunas cactáceas como Ariocarpus fissuratus (Martínez-Peralta y Mandujano, 2011), Escontria chiotilla (Oaxaca-Villa et al., 2006), Ferocactus histrix (del Castillo, 1994) y Opuntia rastrera (Mandujano et al., 1996). El horario de antesis parece estar relacionado con el síndrome de polinización de las especies, especialmente cuando existe algún agente polinizador específico (que es el caso de las cactáceas columnares de antesis nocturna), mientras que las especies que tienen varios polinizadores generalistas o síndromes melitófilos son predominantemente diurnas (Mandujano et al., 1996, 2010; Johnson y Steiner, 2000). Aún se desconocen los polinizadores de $M$. huitzilopochtli; sin embargo, se ha visto abejas y avispas pequeñas como visitantes.

En cuanto a la longevidad floral, la información disponible en las cactáceas indica que predominan las especies con flores de un solo día de vida (Mandujano et al., 2010), como Hylocereus undatus (Valiente-Banuet et al., 2007), uno a dos días como en Opuntia (del Castillo, 1994; Mandujano et al., 1996) o hasta tres días en Ariocarpus fissuratus (Martínez-Peralta y Mandujano, 2011). Si bien las flores de Ferocactus histrix duran activas de 4 a 6 días (del Castillo, 1994), pocas especies tienen flores con un periodo de vida tan largo como Mammillaria huitzilopochtli, con un promedio de 6 a 7 días, y con una longevidad floral que puede alcanzar 25 días. De acuerdo al criterio de Karron (1997) y Orians (1997), la longevidad floral en especies raras, xenógamas y con área geográfica restringida, generalmente puede desarrollar una adaptación hacia flores de larga vida para facilitar la polinización. Sin embargo, no toda la evidencia apunta a este fenómeno; por ejemplo, se ha reportado que la florivoría reduce hasta en $50 \%$ las flores producidas por A. fissuratus, que tienen una longevidad hasta de 3 días (Martínez-Peralta y Mandujano, 2011). También se ha propuesto que en la transferencia de polen, en especies con flores longevas, predominan los polinizadores generalistas, es difícil que se presente una asociación mutualista obligada (Johnson y Steiner, 2000). Es probable que los polinizadores de M. huitzilopochtli sean generalistas, más que una presión selectiva fuerte por parte de florívoros. En las cruzas experimentales efectuadas en el JB y en las flores cuyo estigma estuvo receptivo por más de un día, una vez que se había efectuado la polinización manual al día siguiente, aún estaba receptivo el estigma. Esto puede ser una adaptación ante la falta de polinizadores, ya que si se incrementa el tiempo de receptividad del estigma, incluso cuando las flores ya han sido polinizadas, es más factible que se efectúe e incremente el número de óvulos polinizados en las flores de esta especie; a diferencia de $M$. grahamii, donde la polinización reduce la longevidad floral (Bowers, 2002).

En cuanto al éxito reproductivo, Godínez-Álvarez et al. (2003) mencionan que el número de semillas varía de acuer- 
do a la forma de vida de la cactácea, en las columnares es mayor de 1,000 y en las globosas menores a 100 semillas. La amplia variación en el número de semillas por fruto que se encontraron en Mammillaria huitzilpochtli, a pesar de la elevada longevidad de sus flores, puede deberse a la escasez de polinizadores por estar en condiciones ex situ, aunque el número de semillas de polinizaciones cruzadas es igual a la encontrada en condiciones naturales (Flores Martínez et al., 2010). La dependencia de polinizadores generalistas, con su consecuente menor eficiencia en la transferencia de polen, incrementa la posibilidad de que un polinizador que la visite pase por flores de otras especies antes de que vuelva a visitar una flor de esa especie, con su consiguiente pérdida de polen en la fase de transportación o acarreo (Richards, 1986; Johnson y Steiner, 2000). Algunas especies del género Mammillaria producen muchas semillas, como M. magnimamma con un promedio de 93 semillas por fruto (Valverde et al., 2004), mientras que otras especies como $M$. crucigera producen menos semillas (promedio de 20 semillas por fruto; Contreras y Valverde, 2002), aunque no se menciona el promedio de óvulos por flor en ambos estudios y la transferencia de polen no se ha evaluado en ninguna especie de Mammillaria.

La producción de néctar en Mammillaria huitzilopochtli es escasa, en un porcentaje cercano a la tercera parte de las flores y con cantidad variable de una flor a otra. Si bien algunas especies de cactáceas globosas producen mucho néctar, como Melocactus curvispinus (Nassar y Ramírez, 2004), otras especies producen poco o nulo, como Hylocereus undatus (Valiente-Banuet et al., 2007). La producción escasa o nula de néctar establece dos posibilidades: (1) el polen es la principal recompensa para los polinizadores y el néctar funge una función secundaria $0,(2)$ una estrategia de engaño por el costo energético asociado a la producción de néctar. Este fenómeno es poco estudiado en Mammillaria y representa una fase importante de la interacción con sus visitantes florales.

El número de óvulos por flor (con un promedio de 111) indica que en Mammillaria huitzilopochtli es bajo en relación a otras especies de cactáceas de forma globosa, como Melocactus curvispinus, que tiene entre 350 y 400 óvulos por flor (Nassar y Ramírez, 2004). Pimienta-Barrios y del Castillo (2002) mencionan que el número de óvulos por flor es muy variable, de 150 a 400 en Opuntia ficus-indica, de 388 a 406 en O. robusta, más de 1,000 en Stenocereus queretaroensis y cerca de 7,200 en Hylocereus undatus. En cambio, el número de granos de polen es elevado pero consistente con lo reportado para otras especies en Cactaceae. Pimienta-Barrios y del Castillo (2002) reportan que en el género Opuntia el número de granos de polen por flor varía desde 160,000 en $O$. rastrera hasta 330,000 para $O$. robusta. La relación polen:óvulo de $M$. huitzilopochtli corresponde a la categoría de xenógama facultativa (Cruden, 1977; Dafni, 1992; Kearns e Inouye, 1993). Esta relación de polen:óvulo es muy variable en Cactaceae, por ejemplo de 400:1 en
M. curvispinus (Nassar y Ramírez, 2004), de 400:1 a 800:1 en varias especies del género Opuntia; de 300:1 a 1,050:1 en Pilosocereus, Stenocereus y Subpilocereus (PimientaBarrios y del Castillo, 2002). Dafni (1992) menciona que un número elevado en la relación polen:óvulo está asociado con plantas polinizadas por polinizadores generalistas. Cruden (1977) por otro lado, sugiere que altos valores polen: óvulo indican un sistema de apareamiento xenógamo facultativo, lo cual coincide con lo observado para la especie.

En Mammillaria huitzilopochtli puede presentarse la tendencia evolutiva citada por Orians (1997), en donde la flor ofrece más recursos para atraer polinizadores que las flores vecinas de otra especie. Mammillaria huitzilopochtli lo consigue con una abundante y continua presencia de polen durante varios días en la misma flor y con varias flores abiertas a la vez en la misma planta. Aun así, existe una deficiencia en la transferencia de polen en condiciones naturales, dada la varianza en el número de semillas por fruto e incluso sin semillas (de cero a 120), que puede deberse a la baja fidelidad de los visitantes florales como ocurre con otras especies raras, como en Astrophytum asterias (Strong y Williamson, 2007) y Stenocereus eruca (Clark-Tapia y Molina-Freaner, 2004). Si bien algunas especies de cactáceas globosas, como Melocactus curvispinus, maximizan la posibilidad de reproducción sexual mediante un largo periodo de floración (cercano a un año; Nassar y Ramírez, 2004), en $M$. huitzilopochtli, que tiene un periodo de floración de tres o cuatro meses, es factible que el incremento en eficiencia de reproducción sexual puede alcanzarse por medio de una alta producción de polen.

Mammillaria huitzilopochtli es una especie xenogama facultativa, por lo que requiere de polinizadores que transporten el polen entre flores de diferentes individuos aunque podrían autofecundarse, con el costo reproductivo de una baja producción de semillas. En las cactáceas predominan las especies con sistemas de entrecruza y xenogamia obligada (Gibson y Nobel, 1986; Pimienta-Barrios y del Castillo, 2002; Godínez-Álvarez et al., 2003). A nivel de entrecruza se ha reportado que al menos 20 especies de cactáceas de la tribu Pachycereeae tienen un sistema de apareamiento xenógamo (Alcorn et al., 1961; Suzán et al., 1994; ValienteBanuet et al., 1997; Casas et al., 1999; Cruz y Casas, 2002; Clark-Tapia y Molina-Freaner, 2004; Molina-Freaner et al., 2004; Ibarra-Cerdeña et al., 2005; Méndez et al., 2005; Oaxaca-Villa et al., 2006).

En Mammillaria huitzilopochtli los atributos florales (periodo de floración, longevidad de sus flores), implican la posibilidad de la geitonogamia. Sin embargo, los resultados sugieren que $M$. huitzilopochtli es autocompatible en muy bajas proporciones y xenógama facultativa, posiblemente por depresión por endogamia (Mandujano et al., 1996). Algunas especies globosas han evolucionado desde la xenogamia hacia la autogamia (Nassar y Ramírez, 2004). En especies raras, de baja densidad poblacional, la tendencia es a la 
autocompatibilidad y/o tener reproducción asexual (Orians, 1997). Mammillaria huitzilopochtli no tiene su categoría de rareza por una baja densidad poblacional, sino por tener un área de ocupación reducida y por establecerse en un ambiente específico (especie saxícola). Gaston y Kunin (1997) y Orians (1997) mencionan que especies raras endémicas y con localización muy específica tienen una alta densidad poblacional, que es el caso de M. huitzilopochtli. De acuerdo con estos autores, el efecto que pudiese tener la densidad poblacional en el tipo de sistema de cruzamiento de especies raras casi no se ha estudiado experimentalmente, y los pocos estudios sugieren que la tendencia hacia la xenogamia obligada polinizada por insectos está relacionada positivamente con la densidad poblacional, ya que posibilita que los insectos puedan hacer recorridos entre plantas reduciendo el gasto energético.

\section{Agradecimientos}

El estudio es parte del trabajo doctoral de AFM en la Universidad Autónoma Metropolitana-Xochimilco (UAM-X) bajo la co-dirección de MMS y JG. Al Jardín Botánico Cassiano Conzatti, CIIDIR IPN Oaxaca por las facilidades otorgadas para realizar los experimentos y el préstamo de las plantas de la colección. Permiso de colecta para los frutos por SEMARNAT, México SGPA/DGVS/00615. La investigación fue financiada por los proyectos CGPI-IPN (No. 20070431, 20080718) a AFM, proyectos BBV BIOCON 04-084, CONACyT CB-2006-1 \#62390 y CONACyT CB-2007-01 \#83790 a JG, proyecto 0350 SEMARNAT-CONACyT y PASPA-DGAPA-UNAM a MCM. Agradecemos a I. Rodríguez por apoyo en campo y Jerónimo Reyes por proporcionar las localidades de las poblaciones de Mammillaria huitzilopochtli. G. Manzanero agradece el permiso otorgado por IPN para una estancia de investigación durante el 2012 para concluir este artículo. Agradecemos los comentarios de dos revisores anónimos quienes ayudaron a mejorar significativamente el contenido del manuscrito.

\section{Literatura citada}

Alcorn S.M., McGregor S.E. y Olin G. 1961. Pollination of saguaro cactus by doves, nectar feeding bats, and honey bees. Science 133:1594-1595.

Bowers J.E. 2002. Flowering patterns and reproductive ecology of Mammillaria grahamii (Cactaceae), a common, small cactus in the Sonoran Desert. Madroño 49:201-206.

Casas A., Valiente-Banuet A., Rojas-Martínez A. y Dávila P. 1999. Reproductive biology and the process of domestication of the columnar cactus Stenocereus stellatus in Central Mexico. American Journal of Botany 86:534-542.

Clark-Tapia R. y Molina-Freaner F. 2004. Reproductive ecology of the rare clonal cactus Stenocereus erucain the Sonoran desert. Plant Systematics and Evolution 247:155-164.

Contreras C. y Valverde T. 2002. Evaluation of the conservation status of a rare cactus (Mammillaria crucigera) through the analysis of its population dynamics. Journal of Arid Environments 51:89-102.

Cruden R.W. 1977. Pollen-ovule ratios: a conservative indicator of breeding systems in flowering plants. Evolution 31:32-46.

Cruz M. y Casas A. 2002. Morphological variation and reproductive biology of Polaskia chende (Cactaceae) under domestication in Central Mexico. Journal of Arid Environments 51:561-576.

Dafni A. 1992. Pollination Ecology. A Practical Approach. Oxford University Press, Oxford.

del Castillo R.F. 1988. Fenología y remoción de semillas en Ferocactus histrix. Cactáceas y Suculentas Mexicanas 33:5-14.

del Castillo R.F. 1994. Polinización y otros aspectos de la biología floral de Ferocactus histrix. Cactáceas y Suculentas Mexicanas 39:36-43.

Flores-Martínez A., Manzanero-Medina G.I., Golubov J., Montaña C. y Mandujano M.C. 2010. Demography of an endangered endemic rupicolous cactus. Plant Ecology 210:53-66.

Franco A.C. y Nobel P.S. 1989.Effect of nurse plants on the microhabitat and growth of cacti. Journal of Ecology 77:870-886.

Gaston K.J. y Kunin W.E. 1997. Rare-common differences: an overview. En: Kunin W.E. y Gaston K.J. Eds. The Biology of Rarity: Causes and Consequences of Rare-Common Differences, pp. 12-29, Chapman and Hall, Londres.

Gibson A.C. y Nobel P.S. 1986. The Cactus Primer. Harvard University Press, Londres.

Gigon A., Langenauer R., Meier C. y Nievergelt B. 2000. Blue lists of threatened species with stabilized or increasing abundance: a new instrument for conservation. Conservation Biology 14:402-413.

Godínez-Álvarez H., Valverde T. y Ortega-Baes P. 2003. Demographic trends in the Cactaceae. The Botanical Review 69:173-203.

Ibarra-Cerdeña C.N., Iñiguez-Dávalos L.I. y Sánchez-Cordero V. 2005. Pollination ecology of Stenocereus queretaroensis (Cactaceae), a chiropterophilous columnar cactus, in a tropical dry forest of Mexico. American Journal of Botany 92:503-509.

Jiménez-Sierra C., Mandujano M.C. y Eguiarte L.E. 2007. Are populations of the candy barrel cactus (Echinocactus platyacanthus) in the desert of Tehuacán, Mexico at risk? Population projection matrix and life table response analysis. Biological Conservation 135:278-292.

Johnson S.D. y Steiner K.E. 2000. Generalization versus specialization in plant pollination systems. Trends in Ecology and Evolution 15:140-143.

Karron J.D. 1997. Genetic consequences of different patterns of distribution and abundance. En: Kunin W.E. y Gaston K.J. Eds. The Biology of Rarity: Causes and Consequences of Rare-Common Differences, pp. 174-189, Chapman and Hall, Londres.

Kearns C.A. e Inouye D.W. 1993. Techniques for Pollination Biologists. University Press of Colorado, Niwot.

Kunin W.E. 1997. Introduction: on the causes and consequences of rare-common differences. En: Kunin W.E. y Gaston K.J. Eds. The Biology of Rarity: Causes and Consequences of Rare-Common Differences, pp. 3-11, Chapman and Hall, Londres.

Mandujano M.C., Golubov J. y Huenneke L.F. 2007. Effect of reproductive modes and environmental heterogeneity in the population dynamics of a geographically widespread clonal desert cactus. Population Ecology 49:141-153.

Mandujano M.C., Montaña C. y Eguiarte L.E. 1996. Reproductive ecology and inbreeding depression in Opuntia rastrera (Cacta- 
ceae) in the Chihuahuan Desert: Why are sexually derived recruitments so rare? American Journal of Botany 83:63-70.

Mandujano M.C., Carrillo-Angeles I., Martínez-Peralta C. y Golubov J. 2010. Reproductive biology of Cactaceae. En: Ramawat K.G. Ed. Desert Plants: Biology and Biotechnology, pp. 197230, Springer-Verlag, Berlín.

Mandujano M.C., Montaña C., Franco M., Golubov J. y FloresMartínez A. 2001. Integration of demographic annual variability in a clonal desert cactus. Ecology 82:344-359.

Martínez M.D., López R.F.M., Flores M.A. y Manzanero M.G.I. 2004. Evaluación de técnicas de propagación de Mammillaria oteroi Glass \& Foster. Naturaleza y Desarrollo 2:5-12.

Martínez-Peralta C. y Mandujano M.C.2011. Reproductive ecology of the endangered living rock cactus, Ariocarpus fissuratus (Cactaceae). Journal of the Torrey Botanical Society 138:145-155.

Méndez M., Durán R., Dorantes A., Dzib G., Simá L., Simá P. y Orellana R. 2005. Floral demography and reproductive system of Pterocereus gaumeri, a rare columnar cactus endemic to Mexico. Journal of Arid Environments 62:363-376.

Molina-Freaner F., Rojas-Martínez A., Fleming T.H. y Valiente-Banuet A. 2004. Pollination biology of the columnar cactus Pachycereus pecten-aboriginum in north-western México. Journal of Arid Environments 56:117-127.

Nassar J.M. y Ramírez N. 2004. Reproductive biology of the melon cactus, Melocactus curvispinus (Cactaceae). Plant Systematics and Evolution 248:31-44.

Nassar J.M., Ramírez N. y Linares O. 1997. Comparative pollination biology of Venezuelan columnar cacti and the role of nectar-feeding bats in their sexual reproduction. American Journal of Botany 84:918-927.

Navarro C.M.C. y Flores M.A. 2002. Aspectos demográficos de Echinocereus pulchellus var. pulchellus en el municipio de Chignahuapan, Puebla. Cactáceas y Suculentas Mexicanas 47:24-32.

Oaxaca-Villa B., Casas A. y Valiente-Banuet A. 2006. Reproductive biology in wild and silvicultural managed populations of Escontria chiotilla (Cactaceae) in the Tehuacán Valley, Central Mexico. Genetic Resources and Crop Evolution 53:277-287.

Orians G.H. 1997. Evolved consequences of rarity. En: Kunin W.E. y Gaston K.J. Eds. The Biology of Rarity: Causes and Consequences of Rare-Common Differences, pp. 190-208, Chapman and Hall, Londres.

Pimienta-Barrios E. y del Castillo R.F. 2002. Reproductive biology. En: Nobel P.S. Ed. Cacti. Biology and Uses, pp. 75-90, University of California Press, Berkeley.
Rabinowitz D., Cairns S. y Dillon T. 1986. Seven forms of rarity and their frequency in the flora of the British Isles. En: Soule M.E. Ed. Conservation Biology: The Science of Scarcity and Diversity, pp. 182-204, Sinauer Associates Inc., Sunderland.

Richards A.J. 1986. Plant Breeding Systems. George Allen \& Unwin, Londres.

Rojas-Martínez A., Valiente-Banuet A., Arizmendi M.C., Alcántara-Eguren A. y Arita H.T. 1999. Seasonal distribution of the long-nosed bat (Leptonycteris curasoae) in North America: Does a generalized migration pattern really exist? Journal of Biogeography 26:1065-1077.

Schemske D.W., Husband B.C., Ruckelshaus M.H., Goodwillie C., Parker I.M. y Bishop J.G. 1994. Evaluating approaches to the conservation of rare and endangered plants. Ecology 75:584-606.

SEMARNAT. Secretaría del Medio Ambiente y Recursos Naturales. 2002. Norma Oficial Mexicana NOM-059-ECOL-2001. Protección ambiental-Especies nativas de México de flora y fauna silvestres-Categorías de riesgo y especificaciones para su inclusión, exclusión o cambio-Lista de especies en riesgo.

Strong A.W. y Williamson P.S. 2007. Breeding system of Astrophytum asterias: an endangered cactus. The Southwestern Naturalist 52:341-346.

Steenbergh W.F. y Lowe C.H. 1969. Critical factors during the first years of life of the saguaro (Cereus giganteus) at Saguaro National Monument, Arizona. Ecology 50:825-834.

Suzán H., Nabhan G.P. y Patten D.T. 1994. Nurse plant and floral biology of a rare night-blooming cereus, Peniocereus striatus (Brandegee) F.Buxbaum. Conservation Biology 8:461-470.

Valiente-Banuet A., Arizmendi M.C., Rojas-Martínez A. y Domínguez-Canseco L. 1996. Ecological relationships between columnar cacti and nectar-feeding bats in Mexico. Journal of Tropical Ecology 12:103-119.

Valiente-Banuet A., Rojas-Martínez A., Arizmendi M.C. y Dávila P. 1997. Pollination biology of two columnar cacti (Neobuxbaumia mezcalaensis and Neobuxbaumia macrocephala) in The Tehuacan Valley, Central Mexico. American Journal of Botany 84:452-455.

Valiente-Banuet A., Santos G.R., Arizmendi M.C. y Casas A. 2007. Pollination biology of the hemiepiphytic cactus Hylocereus undatus in the Tehuacán Valley, Mexico. Journal of Arid Environments 68:1-8.

Valverde T., Quijas S., López-Villavicencio M. y Castillo S. 2004. Population dynamics of Mammillaria magnimamma Haworth. (Cactaceae) in a lava-field in central Mexico. Plant Ecology 170:167-184.

Recibido: 24 de agosto de 2012

Aceptado: 10 de diciembre de 2012 